\title{
I I I 3 MAPSE and TAPSE measured by MRI correlate with left and right ventricular ejection fraction and NTproBNP in patients with in dilated cardiomyopathy
}

\author{
Dirk Lossnitzer*, Henning Steen, Stephanie Lehrke, Grigorios Korosoglou, \\ Constanze Merten, Evangelos Giannitsis and Hugo A Katus
}

Address: University of Heidelberg, Heidelberg, Germany

* Corresponding author

from I th Annual SCMR Scientific Sessions

Los Angeles, CA, USA. I-3 February 2008

Published: 22 October 2008

Journal of Cardiovascular Magnetic Resonance 2008, I0(Suppl I):A238 doi:10.I I86/I532-429X-I0-SI-A238

This abstract is available from: http://jcmr-online.com/content/I0/SI/A238

(c) 2008 Lossnitzer et al; licensee BioMed Central Ltd.

\section{Background}

MRI is the current reference-standard for quantification of systolic left and right ventricular (LV/RV-) function. However, the conventional three-dimensional quantitative assessment of LV and RV-ejection fraction (EF), is time consuming and operator-dependant. The mitral/tricuspid annular plane systolic excursion (MAPSE/TAPSE) towards the apex, on the other hand, can be easily and promptly quantified on MR-images and has been previously shown to, correlate with LV- and RVEF and with the prognosis of patients with heart failure in echocardiographic studies. The aim of this study was to compare the value of MAPSE and TAPSE to the conventional LVEF and RVEF assessment and to the well established biomarker NTproBNP in heart failure.

\section{Methods}

Using MRI short axis cine views we assessed RVEF and LVEF by conventional manual tracing of endocardial borders in systole and diastole in 133 subjects (normal subjects $=28$ and patients with dilated cardiomyopathy $(\mathrm{DCM}=105)$ in a $1,5 \mathrm{~T}$ MR-system. MAPSE and TAPSE were measured on a 4-chamber MR-images. NTproBNP levels were quantified using a Roche Diagnostics Kit.

\section{Results}

All parameters showed significant differences between DCMP and normal subjects. LVEDD ( $64 \pm 0.8$ vs. $52 \pm 0.9$ $\mathrm{mm}), \operatorname{RVEDD}(49 \pm 0.8$ vs. $44 \pm 0.8 \%), \operatorname{LVEF}(37 \pm 1.3$ vs.
$67 \pm 1 \%), \operatorname{RVEF}(37 \pm 1.9$ vs. $53 \pm 1.5 \%$ ) as well as MAPSE $(9.3 \pm 1$ vs. $13.5 \pm 0.6 \mathrm{~mm})$ and TAPSE $(18 \pm 1$ vs. $22.1 \pm$ $1 \mathrm{~mm}$ ) were significantly reduced in DCM ( $\mathrm{p}<0,001$ for all). NTproBNP was significantly increased in all patients with DCM $(2706 \pm 564 \mathrm{ng} / \mathrm{l}$, normal value $<450 \mathrm{ng} / \mathrm{l})$. MAPSE and TAPSE correlated well with LVEF and RVEF respectively $(\mathrm{r}=0.64$ resp. $0.52 \mathrm{p}<0.001)$. NTproBNP inversely correlated well with LVEF $(r=-0.763 p<0,001)$ $\operatorname{RVEF}(\mathrm{r}=-0.47 \mathrm{p}<0.0001)$, as well as MAPSE $(\mathrm{r}=-0.52 \mathrm{p}$ $<0.0001)$ and weakly albeit significantly with TAPSE ( $\mathrm{r}=$ $-0.29 \mathrm{p}=0.0043)$. Furthermore the measurement of MAPSE and TAPSE was less time consuming than conventional three-dimensional quantitative assessment of LVEF and RVEF (approximately $30 \mathrm{sec}$ vs. 3-4 $\mathrm{min}$ ) and independent of myocardial to blood contrast.

\section{Conclusion}

MAPSE and TAPSE can be easily assessed on MR-images and are valid parameters for the accurate and fast estimation of LVEF and RVEF. MAPSE and TAPSE correlated well to NTproBNP, however the prognostic value of MAPSE/ TAPSE in heart failure merits further investigation in future longitudinal studies. 